\title{
Ecología política de suburbia: límites y retos del ordenamiento territorial estadounidense
}

\author{
Gian Carlo Delgado-Ramo*
}

Resumen: La concepción del espacio territorial y su ordenamiento definen en buena medida el funcionamiento de una sociedad, sobre todo, en términos de flujos de materiales y energía. En momentos en que el acceso a combustibles fósiles baratos se deteriora y ante la agudización del calentamiento global, se considera útil la reflexión de cómo se han construido territorialmente las sociedades modernas; en particular la de Estados Unidos (EUA), ciertamente la más despilfarradora del planeta. El presente texto parte, por tanto, de revisar la situación energética-ambiental contemporánea, para luego revisar históricamente el caso de Suburbia, un esquema contemporáneo de construcción espacial-territorial de Estados Unidos que ha tenido como punta de lanza la creciente asfaltización y automovilización del país. Sus consecuencias sociales y ecológicas, entre otras, son analizadas. Se concluye con una valorización de alternativas y retos.

Palabras clave: suburbia, calentamiento global, energía, ordenamiento territorial.

\section{Political ecology of suburbia: limits and challenges of the American land use planning}

Abstract: The land use planning conception, defines pretty much a society's performance, above all in terms of energy and material flow. In a time when access to cheap fossil fuels deteriorates and when the phenomena of global warming is becoming deeper, an assessment on how modern societies have been territorially designed and built, is considered useful. This is particularly true for the case of United States, certainly the most wasteful society of the planet. Thus, this paper begins with an evaluation of the current energy-environmental condition in order to proceed to historically review the case of Suburbia; the contemporary spatial-territorial construction scheme of United States that has had its foundations on an increasing advancement of the car culture and the building of streets and highways. Suburbia's social and environmental implications, among others, are reviewed. The paper concludes with an appraisal of alternatives and challenges.

Key words: suburbia, global warming, energy, land use planning.

Recibido: 24.05 .07

Aceptado: 21.07.08

$$
* * *
$$

\footnotetext{
*Universidad Nacional Autónoma de México, México D.F., México. Email: giandelgado@gmail.com
} 


\section{Preámbulo: calentamiento global y los límites del patrón energético imperante}

Los combustibles fósiles constituyen alrededor del $80 \%$ de la energía final consumida a nivel mundial (Ren21, 2008: 9). Véase Imagen. De este porcentaje, la mayor parte del petróleo se emplea en motores de combustión interna destinados al transporte, y el resto en generación de electricidad y en la petroquímica. Un $50 \%$ del carbón se emplea en la generación de energía eléctrica y lo demás en diversas funciones que van desde la industria hasta el hogar. El gas se emplea crecientemente en la generación de electricidad, seguido de cerca, y prácticamente en montos similares, por la industria, el comercio y usos domésticos. La mayoría de la electricidad, o energía secundaría, se emplea en usos industriales (54\%) y doméstico-comerciales (46\%). Es decir, el destino general del petróleo, carbón y gas tiene tres grandes rutas: 1) generación de energía calórica; 2) de energía eléctrica; y 3) motores de combustión interna.

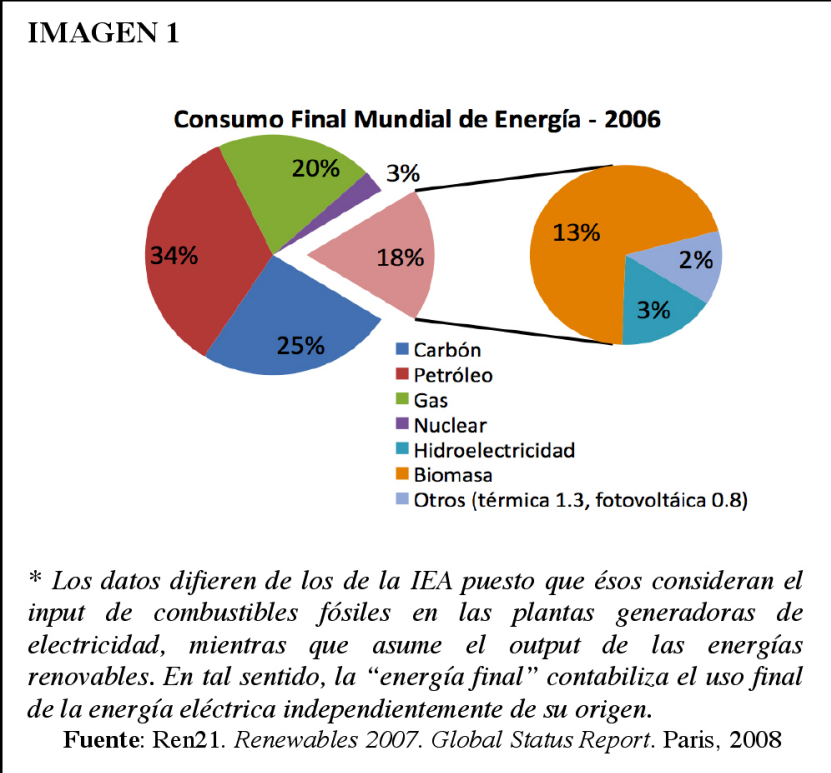

El más relevante, por sus características, es el petróleo. Ello, sobre todo, por el relativo fácil manejo de éste (gracias a sus propiedades físicas) y por el alto grado de condensación (química) de energía en poco espacio. Y es que, incluso comparado con el gas y el carbón, el petróleo permite ventajas únicas de mayor regulación y monopolización de su producción, distribución y almacenamiento; es decir, 
características esenciales en la lucha por la hegemonía regional y mundial. ${ }^{1}$

No extraña entonces que desde su explotación como combustible, el petróleo registre consumos exponenciales, colocándose como la principal fuente energética mundial. Los cálculos indican que se pasó de unos miles de barriles de crudo al año a mediados del siglo XIX, a más de 65 millones de barriles al día para fines del siglo XX (Heinberg, 2003: 92). Sin embargo, como se ha precisado, el gas y el carbón no se quedan atrás pues, como el petróleo, registran índices similares. ${ }^{2}$

El asunto medular está en que el consumo energético es cada vez mayor, tanto por el tipo de sistema de producción establecido, como por el estilo de vida que se fomenta. Ello incluye un peculiar ordenamiento territorial que tiende a promover la concentración de crecientes índices de consumo de materiales y energía, así como de generación de desechos. Y es que las cada vez más grandes y caóticas ciudades, una decena por lo menos en el orden de mega-polis, son por y en principio no-sustentables; más aún, cuando primordialmente éstas son diseñadas para ser funcionales al uso del automóvil y bajo patrones espaciales de exclusión social.

Estimaciones de la Agencia Internacional de Energía (IEA, por sus siglas en Inglés), contemplan un aumento en el consumo energético de alrededor del $57 \%$ en el periodo que va del 2004 al 2030. Esto es, una transición de unos 447 trillones de BTU utilizados en 2004, a 702 trillones de BTU en 2030 (BTU es una unidad de medida del valor de calor del combustible). Se señala que el consumo de gas aumentará a una tasa promedio anual de $1.9 \%$ en el mismo periodo, pasando de 99.6 billones de pies cúbicos en 2004 a 129 billones de pies cúbicos en 2015 y a 163.2 billones en 2030. El carbón, lo haría a un ritmo anual de 2.2\%, es decir, de 14.5 trillones de BTU en 2004 a 199.1 trillones de BTU en 2030 (EIA, 2007).

El consumo energético es sin embargo desigual. Se calcula que los habitantes de los países con mayores ingresos consumen unas 21 veces per capita más que los de bajos ingresos. Cifras a nivel mundial precisan, además, que 2.4 millardos de personas utilizan biomasa tradicional (e.g., madera) para cocinar, mientras que 1.6 millardos no

\footnotetext{
${ }^{1}$ Recuérdese que el gas requiere de energía tanto para su extracción como para su presurización, refrigeración y transportación. El carbón por su parte, tiene un contenido energético menor que el petróleo y, aunque es fácil de transportar, ocupa un espacio comparativamente mayor.

${ }^{2}$ Según estimaciones de 2006 de la Administración de Información sobre Energía de EUA, las reservas de gas a nivel mundial son de poco más de 6 mil billones de $\mathrm{m}^{3}$, siendo Rusia (con 1,688 billones), Irán (943 billones) y Qatar (910 billones) los que acaparan un $60 \%$ de las mismas (www.eia.doe.gov/emeu/international/reserves.html). Es de notarse que en 2005 y 2006 , el consumo de gas supero el producido en esos años lo que indica una tendencia conflictiva entre oferta y demanda (véase: www.eia.doe.gov/emeu/international/gasproduction.html y también www.eia.doe.gov/emeu/international/gasconsumption.html). Más aún, es de considerarse la presión que genera un mayor consumo de gas sobre su precio, pues lo convierte más caro. Ello, a su vez tiende a estimular un uso creciente del carbón.
} 
tienen acceso a la electricidad (Bank Information Center et al., 2006). En plena discrepancia, algunas estimaciones calculan que, en cambio, el conductor estadounidense consume en promedio su peso en petróleo crudo cada semana. Esto dicho de otro modo, significa a nivel mundial, que los automóviles sobrepasan en peso a la población en una relación de 4 a 1 y consumen en combustible una cantidad de energía cercana a esa misma proporción que lo que la gente en alimentos (Heinberg, 2003: 71$)^{3}$

Los datos anteriores son un botón de muestra sobre el hecho de que el grueso del consumo de combustibles fósiles está vinculado a los ingresos y por lo tanto a la variable de clase social puesto que a nivel mundial, según el Banco Mundial, hay alrededor de un millardo de personas sumidas en la pobreza extrema (menos de un dólar al día) y unos 2.5 millardos con menos de dos dólares diarios (www.worldbank.org/poverty). Esto es: la mitad de la población mundial está *prácticamente fuera de los supuestos "beneficios de la modernidad". Por tanto, cuando se habla de patrones intensivos de consumo energético, en buena medida nos referimos al de una fracción de la población mundial; al de unas clases medias y altas que, carentes en general de conciencia socio-ambiental, presiona y avala en su cotidianidad la permanencia de patrones de consumo insostenibles. De ahí que, según la IEA, la demanda global de petróleo para el año 2030 alcanzará un monto de 118 millones de barriles diarios (EIA, 2007).

Se trata de un escenario en el que, por si fuera poco, se está llegando al punto máximo de producción de petróleo convencional "barato", o sea, el de fácil acceso (excluye arenas bituminosas y otras formas de petróleo pesado) ${ }^{4}$; un factor que incrementa no sólo los costes económicos y ambientales de la producción de energía en base a combustibles fósiles, sino también de aquellos relacionados tanto a garantizar el acceso a las reservas, como a velar por la "seguridad" de su extracción y transportación (léase: Delgado y Saxe-Fernández, 2007).

Diversos estudios de geólogos petroleros han calculado que la punta de la campana de producción mundial de petróleo convencional se alcanzará entre 2007 y 2020. Conocida como la "campana de Hubbert", fue calculada por el ingeniero Marion King Hubbert para el caso de EUA, quien estimó que el punto cumbre (peak) se alcanzaría entre 1966 y 1972. El año peak de EUA fue en 1970 aunque no fue visible

\footnotetext{
${ }^{3}$ Actualmente el costo de tener un automóvil se calcula en unos 1,500 dólares anuales, sin embargo, si se suman a éste aquellos aspectos ambientales y sociales, el coste rondaría los 25 mil dólares por automóvil (Heinberg, 2003: 70).

${ }^{4}$ La obtención de petróleo a partir de estas fuentes es extremadamente costoso, tanto en términos económicos y ecológicos. Por ejemplo, hoy en día la producción de petróleo a partir de arenas bituminosas obliga un precio mayor a los 60 dólares por barril para hacerlo viable sin considerar ningún costo ecológico. Y es que el proceso requiere la extracción de dos toneladas de arenas bituminosas para poder obtener un barril de petróleo. El proceso además requiere de 2 de cada 3 barriles obtenidos de estas arenas para cubrir los requerimientos energéticos, miles de litros de agua que luego resultan en 2 y medio barriles de desechos disueltos por cada barril producido (Heinberg, 2003: 127-8).
} 
hasta 1971, de ahí que a principios del siglo XXI, EUA produzca un $40 \%$ menos que lo que hacía en 1970 mientras que su demanda aumentó en un 30 por ciento (HomerDixon, 2007: 86). Dicho en otras palabras, EUA extrae y quema entre 5 y 6 barriles de petróleo por cada uno que es descubierto (Heinberg, 2003: 124). Y la relación aumenta.

Hubbert también estimó que el peak mundial se alcanzaría entre 1990 y 2000, sin embargo muchos de los datos de pozos petroleros que empleó no eran del todo precisos, además de que, desde entonces, la tecnología de extracción posibilitó ampliar ligeramente las reservas probadas de crudo.

Colin J. Cambell, otro geólogo petrolero, ha hecho cálculos actualizados y estima que el peak mundial se tocará entre el 2008 y el 2010, esto es porque considera que el $80 \%$ de los pozos petroleros conocidos fueron descubiertos en 1973, los mismos que en su gran mayoría ya están en fase de declinación (Cambell, 1997). En el mismo sentido, Kenneth Deffeyes habla de un peak de entre 2003 a 2009 (Deffeyes, 2001), mientras que L. F. Ivanhoe, fundador del Hubbert Center for Petroleum Supply Studies en la Colorado School of Mines, coincide al señalar que el peak se alcanzará entre el 2000 y el 2010. Otros, como el geólogo Thomas Magoon del US Geology Survey (USGS) o el Oil \& Gas Journal, son relativamente más optimistas y hablan de un rango de años de entre el 2003 y el 2020 (Heinberg, 2003: 113; DOE, 2004).

En este contexto, es de notarse entonces que, conforme la complejidad de las sociedades modernas incrementa el consumo de energía, aumenta en igual medida la generación de entropía (o aquella energía que ya no puede ser convertida en trabajo); todo, al tiempo que se disminuye la capacidad de carga de los ecosistemas debido a la generación exponencial de residuos. Pero, a diferencia de civilizaciones pasadas, la dimensión y velocidad con que el sistema capitalista de producción depreda los recursos y produce entropía, lo coloca en una situación en la que su propia sobrevivencia es puesta en cuestión (la denominada "segunda contradicción del capitalismo") (O'Connor, 2001).

No sorprende que la huella ecológica mundial, indicador que calcula -en base al actual modo de vida- el espacio territorial necesario, tanto para producir los recursos y energía empleados, como para asimilar los residuos generados por la humanidad, ya sobrepase entre un $25 \%$ y un $39 \%$ al planeta Tierra (dependiendo de los cálculos). ${ }^{5}$ Dicho de otro modo: necesitamos, en el mejor de los casos, un cuarto de planeta adicional para poder mantener los ritmos de consumo y desecho de principios del siglo XXI.

\footnotetext{
${ }^{5}$ Las estimaciones varían. Para la Global Footprint Network, la humanidad pasó de usar, en términos netos, la mitad de la biocapacidad del planeta en 1961 a 1.25 veces en 2003 (Global Footprint Network, 2004). Según Redefining Progress la biocapacidad del planeta había sido rebasada, para el año 2005, en un 39 por ciento (Venetoulis y Talberth, 2005).
} 
Nótese que lo anterior no contempla las eventuales constricciones y efectos de mediano y largo plazo generados por el fenómeno de calentamiento global, el mismo que ya se refleja, por ejemplo, en una exponencial acumulación de gases de efecto invernadero, sobre todo de dióxido de carbono $(\mathrm{CO} 2) .{ }^{6}$ Se trata de una situación que llevó, según la NASA (AFP, 2006), a que la Tierra alcanzara en 2006 su temperatura más alta en 12 mil años; un panorama que, por si fuera poco, promete agudizarse. ${ }^{7}$

Recuérdese que los impactos de corto-mediano plazo de los gases de efecto invernadero, incluyen la contaminación de la vegetación; la filtración de contaminantes a los mantos acuíferos y de ahí al resto de la cadena alimenticia; la lluvia ácida; enfermedades diversas como asma, problemas cardiovasculares, cáncer, irritación y alergias, etcétera (Epsen y Selber, 2002: 35-42). Los impactos de largo plazo, según advierte el Grupo de Expertos Intragubernamental sobre la Evolución del Clima (IPCC, por sus siglas en Inglés), se relacionan con el incremento del número e intensidad de los fenómenos climáticos (tormentas, ciclones, inundaciones, sequías, etc); el desplazamiento y alteración de las reservas de agua dulce; la proliferación de enfermedades provocadas por vectores infecciosos; la pérdida de biodiversidad marina y terrestre; el derretimiento acelerado de los casquetes polares y el consecuente incremento del nivel de los mares y océanos; la alteración de las corrientes marinas (frías y calientes); entre otros (IPCC, 2007).

El panorama anterior es delicado, particularmente para las petroleras, automotrices, llanteras y et al de EUA pues se trata, como se indicó, del país que más consume energía a nivel mundial y, por tanto, del mayor emisor de gases de efecto invernadero (sobre todo en términos per capita; véase imagen 2 para el caso del $\mathrm{CO}_{2}$ ).

No extraña entonces que el gobierno de EUA venga haciendo todo lo posible para facilitar el fluido negocio petrolero, en especial los republicanos, más cercanos a dicho sector y quienes reciben al menos dos terceras partes de las contribuciones petroleras para su activismo político. ${ }^{8}$

Por ejemplo, en 1995, Robert Walker, miembro del Congreso de EUA, logró el apoyo para recortar el financiamiento del programa de la NASA para monitorear los cambios climáticos bajo la argumentación de que se trataba de una investigación poco original cuyos resultados eran más una declaración política que contribuciones científicas (Godrej, 2001: 95). Más haría el republicano Dana Rohrabacher, quién

\footnotetext{
${ }^{6}$ Sólo la cantidad de carbono en la atmósfera, que se mantuvo constante en los últimos 10 mil años en el rango de las 280 partes por millón (ppm), pasó a 360 ppm en 1998 y a 383 ppm en 2006 (Heinberg, 2003: 3).

${ }^{7}$ Según los informes de 2007 del Grupo de Expertos Intragubernamental sobre la Evolución del Clima, de continuar la actual tendencia, se calcula un aumento de la temperatura de entre $+1.8^{\circ} \mathrm{C} \mathrm{y}+4^{\circ} \mathrm{C}$ para el año 2100 de entre un rango mayor que va del $+1.1^{\circ} \mathrm{C}$ a $6.4^{\circ} \mathrm{C}$ (IPCC, 2007)

${ }^{8}$ Según se notifica para los años 1998 y 1997, Mobil Oil dio 84\% de los fondos destinados al apoyo de campañas políticas al partido republicano; Exxon el 88\%; Texaco 76\% y Shell el 74 por ciento (Godrej, 2001: 101).
} 


\section{IMAGEN 2}

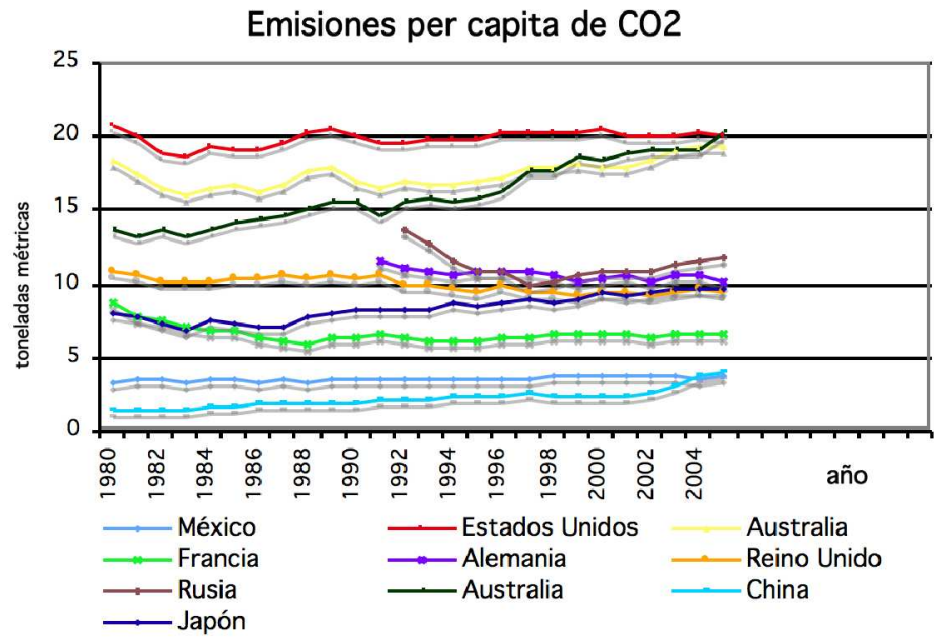

Energy Information Administration. International Energy Annual 2005. Octubre de 2007.

después de publicado el segundo informe del IPCC, señalara: “...pienso que el dinero que se gasta en investigación sobre cambio climático es en realidad dinero tirado a la basura" (Godrej, 2001: 97). Se trata del mismo hombre que en 1995 votó a favor de abrir las zonas prístinas de Alaska a la actividad petrolera y quién también bloqueó el financiamiento destinado a promover la exportación de tecnología solar estadounidense (Godrej, 2001: 97).

Ese tipo de cabildeo se extiende a entes fuertemente financiados por petroleras y afines como lo fue la estadounidense Global Climate Coalition (operativa hasta 2002) y cuyo propósito declarado era "generar duda acerca de la teoría del calentamiento global" (Flannery, 2005: 242). Lo mismo es aplicable para el Competitive Enterprise Institute (EUA), responsable de lanzar una intensa campaña publicitaria que al vincular una caída del estilo de vida estadounidense con el calentamiento global, sostiene que éste no existe. Dicho en sus propias palabras:

...algunos políticos quieren etiquetar ahora el $\mathrm{CO}_{2}$ como un contaminante. ¿Imagina, si tienen éxito, cómo serían nuestras vidas? $\mathrm{CO}_{2}$ : ellos lo llaman contaminante, nosotros lo llamamos vida (www.cei.org).

El fenómeno no es exclusivo de EUA, también se identifica en Australia donde el Lavoisier Group, formalizado en abril de 2000 con fuertes vínculos a la australiana Western Minning Corporation, ha llegado a difundir la idea de que el IPCC es una conspiración diseñada por cientos de científicos que alteran sus resultados con el objeto 
de mantener el financiamiento de sus investigaciones (Flannery, 2005: 224). Desde luego, al ser el IPCC un ente conformado por un esfuerzo conjunto entre las Naciones Unidas y la Organización Mundial de Meteorología, y al operar con cientos de expertos científicos, cientos de evaluadores externos y decenas de delegados de diversos países, el señalamiento de Lavoisier se sale de toda racionalidad, donde además su aseveración no es demostrada. Al respecto, y como bien puntualiza Flannery, vale decir que si bien,

...el escepticismo es un elemento indispensable en la investigación científica, éste, cuando la intención es tergiversar en lugar de aclarar, no puede ser visto más que como engaño (Flannery, 2005: 245).

A contracorriente, vale precisar que el calentamiento global, en buena medida visto como resultado de la acción del hombre, es ya un asunto prácticamente aceptado por el grueso de la sociedad (sobre todo con la publicación de los informes del IPCC de 2007 y los fenómenos meteorológicos recientes, i.e. inundaciones, tsunamis, tornados). Ésta es, sin embargo, una cuestión por lo menos "incómoda" (Gore, 2006) para el empresariado petrolero y afín en tanto que afecta e impone límites a sus intereses cortoplacistas.

Pero también lo es, aunque en diverso grado, para aquella fracción de seres humanos que viven bajo estilos de vida despilfarradores. Me refiero en particular al $20 \%$ de la población mundial que habita en países metropolitanos y que, según se estima, ha generado el $90 \%$ de los gases de efecto invernadero en términos históricos (Godrej, 2001: 95). El caso de EUA y su devastadora suburbia es, en dicho contexto, particularmente ejemplificador.

\section{La conformación de suburbia y sus limites socio-ambientales}

En la historiografía de EUA, el proceso de expansión y ordenamiento territorial tuvo dos ejes operativos: a) el desalojo de comunidades nativas y b) la especulación.

La transferencia comercial de la propiedad como mecanismo distributivo, se convirtió entonces, según aprecia el historiador Howard-Kunstler, “...en la base de la planeación moderna del uso de la tierra en EUA, lo que implica en sí, una escasa sino es que una ausencia total de planeación" (Howard-Kunstler, 1994: 26).

Se trató pues de asumir el espacio geográfico como un recurso privado propio a la "libertad civil" y no como un recurso social. Consecuentemente, advierte el autor, se degradó la noción de que la propiedad individual tiene una responsabilidad con la esfera pública, algo que se reflejó en una tendencia a cuadricular el espacio dejando de lado toda noción integral del espacio colectivo o cívico (Howard-Kunstler, 1994: 27, 30). Esto es que, "....las ciudades estadounidenses florecieron casi solamente como centros de negocios, y lo mostraban. [En el proceso] Los estadounidenses omitieron la 
construcción de espacios ceremoniales y de estructura públicas..., lo que requería el negocio eran oficinas, fábricas, casas para trabajadores y poco más [...] El uso del espacio, del real state, era un resultado inevitable: maximizar el lote de construcción, punto (Howard-Kunstler, 1994: 33).

Así, en momentos en que la industrialización del espacio estadounidense estaba en pleno apogeo, la necesidad por salir de las ciudades se hizo patente pues éstas, entre otras cuestiones, estaban altamente contaminadas (sobre todo por la quema de carbón). El prototipo de suburbio aparecía pues a mediados del siglo XIX. Llewellyn Park estaba conectado a la ciudad de Nueva York por tren o carroza y consistía en una zona exclusiva de residencias sin ningún tipo de espacios vinculantes, sean de trabajo productivo, mercados o instituciones culturales. Mucho menos de un centro o downtown. Además, estaba pensado para una clase social en particular: los acaudalados.

Suburbia poco a poco se fue expandiendo, consumiendo tierras a un ritmo cada vez mayor y por tanto a costa del espacio rural estadounidense. Para principios del siglo $\mathrm{XX}$, aparecían los primeros suburbios de clases medias-altas, un fenómeno que con el tiempo se profundizaría por medio del empuje y socialización del automóvil, la asfaltización del país y la idealización de pertenecer a una clase social diferente que podía darse el lujo de vivir "en medio de la naturaleza".

Así pues, los suburbios se consolidarían en la conciencia social estadounidense, como espacios en los que se cuenta con todas las comodidades citadinas en medio de lo rural. Su rápida y continua proliferación, sin embargo, los concretizaría como lugares sin una economía propia, al tiempo que las ciudades permanecerían como centros de negocios en los que había que colocar, en el menor espacio posible, aquellos trabajadores sin medios suficientes como para acceder a la versión de suburbio de "menor nivel".

El impacto de tal construcción del espacio no tiene precedentes. Particularmente a partir de la década de 1930 se empiezan a registrar cambios significativos en las formas de organización social y su dinámica, en especial en la vida intra-familia y también en el desarrollo urbano, derivados del aumento, persistente y creciente, del uso del automóvil. El fenómeno conoció en los trabajos de los esposos Lynd-Middletown y Middletown in transition- (Lynd y Lynd, 1929 y 1937), el despegue de un vasto esfuerzo de investigación y acervo bibliográfico sobre la "suburbanización", las pautas de trabajo, de recreación, de cortejo, de socialización de niños y jóvenes, con nuevos valores de consumo para el establecimiento de los prestigios y "status" sociales centrados en el automóvil particular (Delgado y Saxe-Fenández, 2007).

El rol del empresariado, pero también del gobierno, fue nodal para abrirle paso al automóvil y todo el negocio asociado, al tiempo que se dificultaba el fortalecimiento del transporte público. El transporte privado fue fuertemente subsidiado mientras que el público (como los tranvías o líneas de camiones) fue altamente regulado de modo tal 
que su existencia en el ámbito privado se fue convirtiendo prácticamente en algo inviable; ello a pesar de que en un principio las empresas de tren ligero se enganchaban en proyectos de emplazamiento de suburbios como su principal negocio. Y es que con el automóvil se logró ampliar el espectro de suburbia al abrir la posibilidad de "rellenar" los espacios vacíos entre las ciudades, los corredores de tranvías, trenes o trenes ligeros y los suburbios ya emplazados.

La asfaltización como mecanismo de reestructuración del espacio para acomodar el automóvil en el centro del estilo de vida estadounidense, fue entonces pagada por toda la sociedad, incluyendo a aquellos que no podían adquirir un automóvil. Las clases medias y altas proclamaron el uso del erario público para asfaltizar al país y promover la expansión del suburbio pues les beneficiaba directamente, aunque claro está, el gran programa de obras públicas abrió cause a un crecimiento económico que se vio reflejado en empleos e incremento relativo de los salarios.

El gobierno federal aprobó la Ley de Carreteras Federales en 1916 con un presupuesto de 75 millones de dólares, al tiempo que impulsaba a los estados a cofinanciar sus propias oficinas para el manejo de carreteras. En 1921 lanzaba una segunda Ley de Carreteras Federales con el fin de mejorar los cerca de 400 mil kilómetros de carreteras estatales a modo de conformar una red nacional de carreteras. Por primera vez en la historia de EUA, el gasto en autopistas alcanzó el millardo de dólares para un solo año: 1925 (Howard-Kunstler, 1994: 90). Como resultado, de 1933 a 1940, las agencias federales gastaron más de 4 millardos de dólares en calles y carreteras (Howard-Kunstler, 1994: 97). Con tal apoyo, el transporte público decayó y muchas compañías quebraron.

La guerra contra el transporte público era además ejecutada por la propia industria automotriz. Como recapitula Howard-Kunstler (1994: 89-92), en 1925, General Motors comenzaba una campaña de compra sistemática de empresas para desaparecerlas o convertirlas en líneas de camiones de su propia marca. Comenzó con la Yellow Coach Company, y continuó al hacerse el accionista mayoritario de Greyhound Corporation. Para 1932, General Motors había constituido la National Highway Transport Corporation -luego la Atlantica Greyhound Lines- para proveer servicio interurbano en el sureste de EUA. En 1932 formaría la United Cities Mobile Transit a partir de adjudicarse empresas de tranvías en ciudades pequeñas y transformarlas en líneas de camiones. Aunque fue disuelta en 1935 por intentar desmantelar las líneas de trolebús en Portland, General Motors continuó su campaña. En 1936 junto con Standard Oil de Califormia y Firestone Tire and Rubber, conformó la compañía National City Lines. Dos años después ésta abría paso a su filial Pacific City Lines y continuaba la compra y desmantelamiento de los sistemas de tranvías y tren ligero en San José, Stockon y Fresno en California. En 1943, otra filial, American City Lines, convirtió los trolebuses a camiones en 90 ciudades más, incluyendo la "gran línea roja" de Pacific Electric 
(fundada en 1887) y que totalizaba unos $2,570 \mathrm{~km}$. lineales a lo largo y ancho de Los Ángeles. Para 1950, añade Howard-Kunstler, General Motors y compañía habían convertido más de 100 líneas de tranvías a camiones. El camión, puntualiza, era la excusa para romper el esquema de transporte eléctrico y abrir en el mediano-largo plazo todo el espacio al automóvil privado (Howard-Kunstler, 1994: 89-92, 208-216).

Para ese entonces, la expansión de suburbia parecía llegar a sus límites. Las carreteras existentes ya no podían manejar un número mayor de tráfico si la construcción de suburbios continuaba desenfrenadamente. La solución fue la ampliación del sistema de carreteras. El presidente Dwight D. Eisenhower (1953-1961), con todo el apoyo de la industria automotriz-petrolera, lanzó para tal propósito la Ley de Ayuda Federal a las Carreteras de 1956. El comité presidencial conformado para la elaboración de dicha iniciativa de Ley había sido presidido por Lucius D. Clay quien también era parte del consejo de directores de General Motors (Howard-Kunstler, 1994: 106).

La medida planteada fue entonces justificada y asumida como una acción de seguridad y defensa nacional, pues se trataba de canalizar fondos federales (en un 90\%) a la construcción de una vasta red de superautopistas semejante al "Autobahn" construido por Hitler (Heinberg, 2005: 70). Conocida como Sistema Inter-Estatal de Autopistas (Interstate Highways System, IHS), es un extenso tejido que en 2004, con una inversión acumulada de más de 130 mil millones de dólares, contaba con 75, 376 kilómetros de extensión y llegaba a todos los principales centros urbanos.

El IHS, una infraestructura adicional al de por sí inmenso Sistema Nacional de Carreteras antes descrito, contrasta con sus contrapartes en otros países industrializados, pues su diseño incluye el paso por los centros urbanos (downtown) de asentamientos de más de 50 mil habitantes. Además incluyó la construcción de cinturones carreteros alrededor de las grandes urbes del país. Ello, fortaleció aún más y como nunca antes el uso del automóvil y el desarrollo suburbano de posguerra a un costo de miles de toneladas de acero, concreto, cobre y demás materiales (Howard-Kunstler, 1994; Cox y Love, 1996). Las estimaciones sugieren un ritmo de consumo anual de materiales equivalente al de 100 ciudades, cientos de cuadrillas de maquinarias y cientos de miles de trabajadores. A decir de Howard-Kunstler, "... simplemente fue el proyecto de obra pública más grande de la historia del mundo” (Howard-Kunstler, 1994: 107). Y es que el IHS fue pensado para uso tanto civil como militar ya que su diseño estructural permite el traslado de una amplia gama de abastecimientos y maquinaria de guerra incluyendo la muy pesada, municiones, tanques y la movilización frecuente -y manejode cohetería balística intercontinental (Delgado y Saxe-Fernández, 2007). Es una noción que permitió mantener su desarrollo aún con el empantanamiento de EUA en la guerra de Vietnam (1959-75). 
Para los primeros años de la década de 1970, el proyecto era culminado. Las nuevas supercarreteras ampliaban la capacidad expansiva de suburbia. Los desarrollos inmobiliarios, o mejor dicho, la "monocultura inmobiliaria" y toda la proliferación de centros comerciales y tiendas de conveniencia a lo largo de las autopistas no se hizo esperar. Un nuevo ciclo expansivo del sector inmobiliario era abierto con todo el apoyo gubernamental del gobierno de Reagan y los gobiernos siguientes. Sus consecuencias, tan sólo en términos de especulación financiera, tuvieron su primera aparición con la crisis inmobiliaria de la década de 1980. A principios del siglo XXI, una segunda edición aún mucho más aguda se viene gestando con repercusiones que en buena medida aún son impredecibles.

Ahora bien, en términos energéticos, las implicaciones del ordenamiento territorial estadounidense son agudas, y sus límites evidentes. Datos de 2003 precisan que EUA consumió 20 millones de barriles diarios en promedio, de los cuales dos tercios se destinaron sólo al sector transporte (Hirsch et al., 2005: 4). Los automóviles suman en ese país unos 130 millones y consumen el $25 \%$ de petróleo quemado en ese país (4.9 millones de barriles diarios); los camiones ligeros (vans, SUVs) son unos 80 millones y totalizan el $18 \%$ del petróleo (3.6 millones de barriles diarios); los camiones pesados o de carga, son unos 7 millones y absorben el 16\% del petróleo ( 3 millones de barriles diarios). Se suma una flota de 8,500 aviones que devoran el 6\% del petróleo o 1.1 millones de barriles diarios (Hirsch et al., 2005: 23).

Lo anterior contrasta con datos mundiales, pues mientras en el orbe sólo el 8\% tiene automóvil, en EUA, el 89\% tiene por lo menos uno (Godrej, 2001: 124). Su uso es además intensivo, de ahí que se calcule que EUA quemó en 2007 cerca de la mitad de los 1,300 millardos de litros de gasolina consumidos mundialmente (Ren21, 2008: 6).

Es claro que suburbia es altamente devastadora. Además de sus enormes costos sociales, que por cierto hemos ignorado, escribe Howard-Kunstler, "...la totalidad del sistema de expansión suburbana es demasiado cara de operar, es muy costosa de mantener y es una amenaza a la ecología..." (Howard-Kunstler, 1994: 114).

Y lo que es más, el despilfarro estadounidense no sólo se limita a la cuestión energética, sino a todos los rubros. En agua, el estadounidense promedio consume por lo menos dos veces más que una persona de la periferia con acceso a ésta; y en esa misma proporción genera basura comparado con sus contrapartes de España, Italia o Alemania, ya no se diga de otros países periféricos (Godrej, 2001: 124). La expansión de los costos socioambientales de, por ejemplo, una cena estadounidense promedio se desborda incluso hasta dos mil kilómetros donde fueron producidos algunos productos (Godrej, 2001: 124). El esquema tan solo energéticamente hablando es insostenible en el mediano-largo plazo. 


\section{Alternativas a suburbia}

Destacan dos acciones clave frente a tal caótica concepción del espacio. Por un lado, la inminentemente necesaria "des-automovilización" por la vía de un aumento del transporte público de calidad (lo que no significa, por lo menos en el corto-mediano plazo, la negación total del automóvil). Por el otro lado, la reconfiguración del territorio por la vía de diseños arquitectónicos económica y energéticamente más eficientes y menos agresivos psicológicamente hablando (se ha construido un espacio público compuesto en su gran mayoría por calles, carreteras y espectaculares luminosos, un ámbito en el que usualmente el automovilista tiene toda prioridad; aunque, claro está, se suman los centros comerciales con sus grandes zonas de estacionamiento como espacios de socialización o de "comunidad").

El nuevo urbanismo ha sido propuesto desde la década de 1970 como crítica a las mencionadas discontinuidades e irracionalidades arquitectónicas que promueve el modernismo en tanto a la funcionalidad integral del espacio. Para tal corriente, se asume la necesidad de una conectividad en base a una conciencia emocional y psicológica profunda de los patrones comunes de los espacios, tanto públicos como privados. La concepción del espacio es pues reformulada para proveer esquemas "integrales verdes". Esto es, comunidades compactas y de largo tiempo de vida que utilizan diseños, materiales y tecnologías verdes (para el ahorro energético, de agua u otros recursos, así como para la disminución de desechos), de usos múltiples (habitacional, recreativo, comercial, etcétera), con espacios verdes comunes y privados y áreas públicas funcionales tanto en términos de acceso como de disfrute (diseño estético del espacio arquitectónico). El automóvil deja de ser el medio de transporte interno central y pasa a uno secundario siendo éste sustituido por una diversidad de medios, desde la caminata corta, hasta el uso de bicicletas y medios de transporte público impulsados por energía eléctrica.

Ya hay iniciativas que exploran en la práctica dicha modalidad de "nuevo urbanismo". En EUA, están las de Seaside en Florida (el precursor clásico que comenzara a tomar cuerpo en 1981), la de Haile Village Center en Gainesville (Florida), la de Harbor Town en Memphis (Tennessee), la de Addison Circle (Texas), la de Orenco Station en Hillsboro (Oregon), la de Mashpee Commons (Massachusetts) o la de Celebration en Orlando (Florida). Si bien varían sus características y grado de funcionalidad ecosocial real, en general todos los casos tienen como meta normativa, romper con el modelo de ciudad o de asentamiento humano como máquina devoradora de tierra, energía y agua, y que al mismo tiempo desecha inmensas cantidades de todo tipo de contaminantes. Dicho de otra manera, se trata de un modo operativo que, particularmente para el caso estadounidense, se aleja de la idea de que los espacios habitacionales sólo han de asociarse con calles (no necesariamente con camellones y banquetas), autopistas y cables eléctricos y de fibra óptica. Desde luego hay contradicciones importantes en el grueso de proyectos del nuevo urbanismo, en especial en el sentido de que siguen siendo comunidades excluyentes puesto que son pensadas, 
diseñadas y estructuradas para clases sociales de altos ingresos económicos. Este rasgo coloca a dichos proyectos como esfuerzos de rediseño material-espacial de suburbia; pero no en un sentido sociocultural amplio e integrador (véase más adelante).

El nuevo urbanismo, aunque propone ciertos aspectos positivos, al mismo tiempo puede ser funcional para la complejización del problema de suburbia en los sentidos arriba indicados, pero también respecto a sus dimensiones. Y es que los proyectos de expansión de suburbia fuera de las fronteras estadounidenses y hacia zonas de playa transfronterizas, parece ser un patrón cada vez más repetitivo en las costas mexicanas; especialmente en la Península de Baja California y la Riviera Maya. Por ejemplo, el Monarch Cancún es un proyecto "integral" actualmente en proceso de desarrollo en un área prístina de 1,820 hectáreas localizada a 32 kilómetros del aeropuerto de Cancún. Incluye ocho mil lotes de mil metros cuadrados por un precio de 150 mil dólares más costos de construcción, tres campos de golf de 18 hoyos, SPAs, un rodeo-centro cultural, canchas de tenis, club de playa, restaurantes, centros de entretenimiento, un centro comercial y de negocios, un centro ecuestre y establos privados, una zona de horticultura, invernaderos, entre otros espacios (www.monarchcancun.com). Dirigido al mercado de jubilados estadounidenses, es impulsado por el Grupo ICA (en especial por Aramburozabala y Quintana Isaac) y E-Sol International (que opera mediante International Country Club Corp., JP Capital Corp., y Electrificación y Proyectos, S.A.). Se trata de las iniciativas más recientes en la Rivera Maya, pero ciertamente no la única (Conde, 2008).

"Villas de Bahía de Loreto", en el Mar de Cortez, es otro caso que es desarrollado específicamente bajo la rúbrica del nuevo urbanismo. Al parecer, se trata del asentamiento de ese tipo, más grande del continente y que, al mismo tiempo, es una de las piezas claves de la Escalera Náutica ${ }^{9}$ Se extiende en una línea costera de $7 \mathrm{~km}$ con una superficie de 3.2 hectáreas de reserva natural en Nopoló, Baja California Sur. Ahí se construyen 6 mil casas-habitación de diseño y materiales verdes (como adobe térmico). Se suma un club de playa, una marina, SPAs, dos campos de golf de 18 hoyos, restaurantes, galerías de arte, un centro médico y otro comercial, un mercado, entre

\footnotetext{
99 La "Escalera Náutica" es un proyecto añejo que fue ideado por Guillermo Rosell, titular de Turismo en el gobierno de José López Portillo (1976-82), quien trató de llevarlo adelante y no lo logró. Fue reactivado por Vicente Fox (2006-06) en febrero de 2001 cuando se firmó un convenio entre la presidencia, la Secretaría de Turismo, el director del Fondo Nacional de Fomento al Turismo - Fonatur (John McCarthy) y los gobernadores de los estados involucrados. Con una inversión pública-privada de al menos unos 2,250 millones de dólares, incluye el desarrollo de 24 escalas náuticas, 11 centros náuticos, un puente terrestre, aeropuertos y rutas de internación para embarcaciones remolcables a lo largo y ancho de la península de Baja California y las costas de Sonora y Sinaloa (para mayores referencias, véase: www.escaleranautica.com). Se suman millonarias inversiones en infraestructura hotelera y turística. Por ejemplo, tan sólo en el área que va de Tijuana a Ensenada, se calcula que al año 2006 había unas seis decenas de proyectos inmobiliarios en desarrollo que totalizaban más de 11 mil casas-departamentos y un valor por encima de los 3 millardos de dólares. Todos enfocados a potenciales compradores extranjeros, sobre todo estadounidenses (Davis, 2006).
} 
otras instalaciones relacionadas al tema de agua y energía como lo son las plantas de tratamiento de agua y de manejo de residuos o la granja eólica de 20MW (con planes de triplicar su capacidad) (Ramírez, 2007; www.loretobay.com.mx). El proyecto es impulsado por la Compañía de Bahía de Loreto, una subsidiaria del Trust for Sustainable Development de Canadá, y por la Fundación Bahía de Loreto que es controlada por The Ocean Foundation de EUA y en la que entre sus miembros del consejo figuran personajes de IUCN, National Geographics, Rockefeller \& Co.,entre otros entes (www.oceanfdn.org/index.php?tg=articles \&topics=29). Los socios empresariales son múltiples y el negocio es millonario (de hasta unos 3 millardos de dólares) (Davis, 2006). En pleno proceso de conformación, Villas de Loreto ya ha invertido unos 200 mdd y ofrece casas o departamentos que van, en promedio, de los 370 mil dólares al millón y medio de dólares. El grueso de clientes: estadounidenses y canadienses millonarios o jubilados (Ramírez, 2007).

Nótese que el creciente fenómeno de migración-a-la-inversa, es decir de baby boomers que van a gozar sus jubilaciones de oro en México, contrasta de modo importante con la naturaleza y dimensiones de la histórica migración de trabajadores mexicanos hacia EUA. Y es que mientras se fortalecen las políticas antimigratorias y se construye un muro transfronterizo, también se ha facilitado el emplazamiento de "extensiones" del suburbio estadounidense (y canadiense) en México. La especulación inmobiliaria de lujo, que usualmente involucra la ocupación y privatización de facto de costas y tierras ejidales, de zonas prístinas y sus diversos recursos, así como el desalojo de población local, ha llevado a que el número de migrantes-propietarios estadounidenses en el país, pasara de unos 200 mil en 1996 a más de un millón en 2006 (equivalente a un cuarto de todos los expatriados de EUA) (Davis, 2006). Por tanto, aunque con sus rescatables avances, el nuevo urbanismo, sigue siendo contradictorio y limitado pues estimula la atomización de la gente al desalojar y excluir a "unos" mediante la apropiación de un espacio que, por tanto, debe ser privatizado y protegido de los "otros". Si bien el diseño material se mejora, la cultura energética y de agua detrás de tales proyectos netamente turístico-derrochadores, resulta altamente contradictoria.

Considerando lo indicado y de frente al esperado aumento de la población urbana en las próximas décadas -se estima que sólo África y Asia verán un aumento de su población urbana de alrededor de 1,800 millones de personas más para el $2050{ }^{10}$, debe precisarse que la alternativa más refinada está entonces en la construcción material pero también socio-cultural y psicológica del espacio como reflejo de una comunidad

\footnotetext{
1010 A principios del siglo XXI, la población urbana cubre un área del 3\% de la tierra y está concentrada, en tres cuartas partes, en 25 países. El incremento de ésta, en el orden de 1,800 millones de personas para el 2050 en África y Asia, responde en buena medida al inusitado ritmo de crecimiento de la población urbana china e hindú. China cuenta hoy en día con una población $40 \%$ urbana, es decir, de 500 millones de individuos. Para el 2050 se espera que ésa lo sea en un $70 \%$ o mayor a mil millones de personas. India tiene una población $30 \%$ urbana o de 300 millones, la misma que se calcula que aumentará a un $55 \%$ o 900 millones para el 2050 (ONU, 2008).
} 
orgánica, multicultural y diversa, e incluso, de tipo urbano-rural. Esto último es fundamental pues cada vez más aumenta la cantidad de kilómetros detrás de los alimentos que consumimos lo que, consecuentemente, incrementa su "mochila ecológica" (Watkiss et al, 2005). La medición de los kilómetros detrás de los alimentos (o la contabilidad de food miles) da una buena idea del fuerte input energéticoambiental que no es contabilizado. ${ }^{11} \mathrm{Y}$ es que si tan sólo esos aspectos se consideraran, digamos en términos de la mochila de $\mathrm{CO}_{2}$ que cada alimento lleva consigo, los alimentos importados se encarecerían. ${ }^{12}$

Lo anterior, lejos de ser una medida proteccionista (como algunos la califican) (Saunders et al, 2006), responde a la necesidad de fomentar una aproximación más sustentable de la economía, y mejor aún, de la sociedad humana y su organización espacial-productiva. Se trata de una noción que efectivamente se antepone al entendimiento capitalista de "globalización" y que, entre otras cosas, implica la destrucción de las economías locales y de las relaciones productivas, sociales y culturales que éstas generan. Nótese que la organización del espacio refleja el hecho de que "la comunidad", altamente atomizada, cada vez sabe menos cómo conectarse si no es por medio del automóvil, los teléfonos o el internet.

La observable y creciente pérdida de la capacidad humana de socialización nomediada y de organización colectiva es una cuestión delicada en todos los sentidos, incluyendo meramente el de eficiencia energética. Por lo tanto, el proceso de transformación hacia una nueva modalidad de configuración del espacio ecosocialmente viable, como bien concluye Howard-Kunstler, requiere, entre otros aspectos, de:

...reducir la escala de nuestras gigantescas empresas e instituciones...y aprender a vivir a escala local y por tanto responsablemente. Tendremos que conducir menos y crear un sistema de transporte público decente que la gente quiera usar. Tendremos que

\footnotetext{
${ }^{11}$ Ciertamente el número de kilómetros no contabiliza la totalidad de la energía empleada detrás de nuestros alimentos pues a ello habría que sumar la energía utilizada durante su proceso de producción y empaquetamiento. Incluso, habría que sumar la energía utilizada para el reciclaje de la basura generada (como plásticos y demás embalajes).

${ }^{12}$ La lógica capitalista de producción de principios del siglo XXI es profundamente irracional y en buena medida sólo posible gracias al acceso a crecientes fuentes de energía barata. Esto es claro por ejemplo en las balanzas comerciales de varios países en las que se registran importaciones y exportaciones de los mismos productos (caso del Reino Unido en lo que respecta a la leche: en 1997 exportó 126 millones de litros de leche y 23 mil toneladas de leche en polvo pero, al mismo tiempo, importó 270 millones de litros y 153 toneladas de leche en polvo) (Saunders et al, 2006: 3). A ello se suma la constante operativa de "aprovechamiento de las ventajas comparativas" de cada región que en muchos casos incrementa el consumo energético. Por ejemplo, en el caso de los alimentos, el grueso del bacalao noruego es enviado a Asia para su empaquetamiento y luego devuelto o enviado a otros destinos de exportación. La razón de tal movimiento es que la mano de obra noruega equivale a 2.70 dólares por kilo, mientras que en Asia no supera los 50 centavos. El costo ambiental de su transportación no es desde luego contabilizado más que en cuanto al gasto de combustible y manejo de cargo (Rosenthal, 2008).
} 
generar menos basura (incluyendo la contaminación atmosférica) y consumir menos combustibles fósiles... (Howard-Kunstler, 1994: 275).

Desde luego un marco legal-jurídico ad hoc es fundamental para estimular dicho cambio. Por ejemplo mediante legislaciones que incentiven el desarrollo y uso de energías y demás tecnologías alternativas; que obliguen la planeación integral y sustentable del espacio local, regional y nacional (al estilo de comunidades de ecoaldeas); que limiten las dimensiones de los asentamientos urbanos; que obliguen la implementación de técnicas de "bioconstrucción", que descentralicen las diversas actividades económicas; que incentiven la agricultura urbana de autoconsumo (jardines y pequeños cinturones de producción urbanos de, por ejemplo, hortalizas); que estimulen el emplazamiento de sistemas de transporte públicos de buena calidad, incluyendo ciclovías de barrio; que por lo menos exijan la venta de autos híbridos y por tanto de alto rendimiento energético; que limiten el uso del automóvil, comenzando, por ejemplo, con la aplicación de impuestos a la compra y uso privado de automóviles de alto caballaje y de dimensiones cúbicas considerables (puesto que requieren de una asignación mayor de espacio público -calles y carriles más amplios); etcétera.

Aún así, ciertamente hay que reconocer que, mientras la lógica del sistema de producción sea el crecimiento económico, el total del consumo energético y de materiales nunca será menor, aún cuando se logren índices crecientes de eficiencia. De ahí pues que se asuma necesario hablar de la necesidad de redefinir la lógica del sistema de producción hacia el decrecimiento económico; lo que no implica un decrecimiento de la calidad de vida cultural, psico-emocional, físico-intelectual y ético-social de la población, sino lo contrario. Por ejemplo, medidas que reduzcan el consumismo de embalajes y empaques, pero también de otros productos que actualmente llegan a su punto de vida útil final de modo crecientemente más rápido. Desgraciadamente ello implicaría, en el largo plazo, la desaparición de buena parte del consumismo basado en la moda, entre otras cuestiones. Si bien ello ocasionaría pérdidas a ciertas empresas, ocasionando un decrecimiento, la calidad de vida bien podría aumentar, sobre todo en lo que refiere a lo ecosocial. Lo anterior no implica por tanto que la gente deba restringir sus necesidades, ni mucho menos, simplemente sugiere dejar de lado un estilo de vida que se sustenta en despilfarrar recursos finitos con el principal y a veces único objeto de que algunos puedan hacer potenciales y jugosos negocios. El problema de fondo es estructural pues en tal caso ya no estaríamos hablando de un sistema de producción capitalista, al menos en los términos en los que actualmente se concibe. 


\section{Bibliografía}

AFP (2006), "Alcanza la Tierra su temperatura más alta en 12 mil años: NASA", La Jornada, México, 26 de Septiembre.

Bank Information Center et al. (2006), How the World Bank's Energy Framework Sells the Climate and Poor People Short, EUA, Septiembre.

Cambell, Colin (1997), The Coming Oil Crisis, Multi-Science and Petroconsultants, EUA.

Conde Olivares, Mauricio (2008), "Megaproyecto turístico amenaza los mantos freáticos de Cancún”, La Jornada, México, 28 de abril.

Cox, Wendel Cox y Love, Helen (1996), 40 Years of the US Interstate System: an Analysis. American Highway Users Alliance, EUA, junio.

Davis, Mike (2006), "Border Invaders. The Perfect Swarm Heads South", Tom Dispatch. EUA, 19 de septiembre.

Deffeyes, Kenneth S. (2001), Hubbert's Peak: The Impeding World Oil Shortage. Princenton University Press. EUA.

Delgado, Gian Carlo y Saxe-Fernández, John (2007), "Engaños Contables de los Monopolios de la Energía: costos, impactos y paradigmas del sector", DELOS. Revista Electrónica de Desarrollo Local Sostenible, Vol. 1. № 0, EumedNet, Universidad de Málaga, España, octubre.

DOE - Departament of Energy (2004), "Strategic Significance of America's Shale Oil Resource", Assessment of Strategic Issues, Vol. 1. Office of Deputy Assistant Secretary for Petroleum Reserves, Office of Naval Petroleum and Oil Shale Reserves, EUA, Marzo.

EIA - Energy Information Administration (2007), International Energy Outlook 2007, Paris.

Epstein, Paul R. y Selber, Jesse (eds.) (2002), A life cycle analysis of its health and environmental impacts, The Center for Health and the Global Environment. Harvard Medial School, EUA, Marzo.

Flannery, Tim (2005), The Weather Makers, Penguin, Londres.

Global Footprint Network (2004), Humanity’s Footprint 1961-2003, EUA.

Godrej, Dinyar (2001), No-Nonsense Guide to Climate Change, Verso ,Reino Unido. 
Gore, Al (2006), Una verdad Incómoda, Gedisa, España.

Heinberg, Richard (2003), The Party's Over. Oil, war and the fate of industrial societies, New Society Publishers, Canadá.

Hirsch, Robert et al (2005), Peaking of World Oil Production: impacts, mitigartion \& risk management, EUA, febrero.

Homer-Dixon, Thomas (2007), The Upside of Down, Vintage, Canadá.

Howard-Kunstler, James (1994), The Geography of Nowhere, Touchstone, Nueva York, EUA.

IPCC (2007), Impacts, Adaptation and Vulnerability, Working Group II Report, Ginebra, Suiza, abril.

Lynd, Robert S., y Lynd, Helen M (1929), Middletown: a study in modern American culture, Harvest Book, EUA.

Idem (1937), Middletown in transition: a study in cultural conflicts, Harvest / HBJ Book, EUA.

O’Connor, James (2001), Causas naturales. Ensayos de marxismo ecológico, Siglo XXI, México.

ONU - Organización de las Naciones Unidas (2008), "Press Conference on World Urbanization Prospects", Departamento de Información Pública, Nueva York, EUA, 26 de febrero.

Ramírez, Karla (2007), "Impulsan turismo sustentable en BC", Reforma, México, 1 de marzo.

Ren21 (2008), Renewables 2007. Global Status Report, Paris.

Rosenthal, Elisabeth (2008), "Environmental Cost of Shipping Groceries Around the World", The New York Times, EUA, 26 de abril.

Saunders, Carolina; Barber, Andrew; Taylor, Greg (2006), Food Miles Comparative Energy/Emissions of New Zeland's Agriculture Industry, Research Report No 285, Lincon University, Nueva Zelanda, julio.

Venetoulis, Jason y Talberth, John (2005), Ecological Footprint of Nations. Update 2005, Redefining Progress, EUA.

Watkiss, Paul et al (2005), The Validity of Food Miles as an Indicator of Sustainable Development, Final Report ED50254, Reino Unido, julio. 